\title{
Factors Associated with Disease Stabilization of Desmoid-Type Fibromatosis
}

\author{
Yongsung Kim, $\mathrm{MD}^{*}$, Mamer S. Rosario, $\mathrm{MD}^{*, \dagger}$, Hwan Seong Cho, $\mathrm{MD}^{\ddagger}$, Ilkyu Han, MD ${ }^{*, \S}$ \\ ${ }^{*}$ Musculoskeletal Tumor Center, Seoul National University Cancer Hospital, Seoul, Korea, \\ ${ }^{\dagger}$ East Avenue Medical Center, Quezon City, Philippines, \\ ${ }^{\ddagger}$ Department of Orthopaedic Surgery, Seoul National University Bundang Hospital, Seongnam, \\ ${ }^{\S}$ Department of Orthopaedic Surgery, Seoul National University College of Medicine, Seoul, Korea
}

Background: Spontaneous disease stabilization of desmoid-type fibromatosis (DF) has been demonstrated in many reports, and the watchful waiting approach without any frontline treatment is becoming popular as an initial management strategy. In this study, we aimed to assess the disease stabilization rate and identify predictive factors for disease stabilization of DF in patients with conservative treatment.

Methods: We reviewed 76 patients with sporadic extra-abdominal DF who were managed with frontline conservative treatment in our institute. The minimum follow-up was 12 months. Stabilization was defined as radiological evidence of no change or continuous decrease in size of the tumor for six months or more. The primary endpoint was stabilization of DF. Possible patient-, disease-, and treatment-related factors predictive of disease stabilization were analyzed with multivariate analysis.

Results: At final follow-up, 54 of the 76 tumors (71\%) were stable, and mean time to stabilization was 30.4 months (range, 7 to 112 months). On Kaplan-Meier survival analysis, the spontaneous stabilization rate was $25.4 \%$ at one year, $52.7 \%$ at two years, and $70.9 \%$ at three years. The mean time to spontaneous stabilization was longer in patients with $\leq 40$ years of age $(p=0.022)$ or recurrence $(p=0.041)$. On multivariate analysis with the Cox proportional hazard method, recurrence (hazard ratio $[\mathrm{HR}], 1.79 ; p=$ $0.041)$ and younger age (HR, 2.04; $p=0.022)$ were identified as independent prognostic factors for longer time to disease stabilization.

Conclusions: Frontline conservative treatment seems to be the optimal treatment for most patients with DF. Younger patients or those with recurrence may require longer time to spontaneous disease stabilization.

Keywords: Desmoid, Fibromatosis, Aggressive, Stabilization, Watchful waiting

Desmoid-type fibromatosis (DF) is a rare mesenchymal tumor, which was first described by MacFarlane in $1832^{1)}$ and termed desmoid tumor by Mueller in 1838. This neoplasm is considered benign but locally infiltrative and can occur in nearly any part of the body. ${ }^{2)}$ It occurs sporadi-

Received April 9, 2018; Accepted July 18, 2019

Correspondence to: Ilkyu Han, MD

Musculoskeletal Tumor Center, Seoul National University Cancer Hospital and Department of Orthopaedic Surgery, Seoul National University College of Medicine, 101 Daehak-ro, Jongno-gu, Seoul 03080, Korea

Tel: +82-2-2072-0682, Fax: +82-2-764-2718

E-mail: hik19@snu.ac.kr cally but its association with familial adenomatous polyposis is well known. ${ }^{3)}$ Despite being non-metastasizing, this neoplasm is reported to have a local recurrence rate of $20 \%-64 \%$ after surgical resection, and repeated surgery often results in substantial morbidity. ${ }^{4,5}$ Nonsurgical modalities such as chemotherapy, radiotherapy, and hormonal therapy have been considered an alternative treatment option. ${ }^{6-11)}$ Some authors, on the other hand, advocate a more conservative approach, which is known as "wait-and-see" strategy, as an initial approach. ${ }^{4,5,10-16)}$

The biological behavior of DF is unpredictable and varies widely from indolent to rapidly progressive. ${ }^{17)}$ Meanwhile, spontaneous stabilization of this neoplasm 
Kim et al. Spontaneous Stabilization of Desmoid Fibromatosis

Clinics in Orthopedic Surgery • Vol. 12, No. 1, $2020 \bullet$ www.ecios.org

has been reported in many studies. ${ }^{4,5,18-20)}$ To the best of our knowledge, only one report ${ }^{19)}$ has investigated possible predictors of spontaneous stabilization of sporadic extra-abdominal DF and the size of the cohort was small. In this study, we sought to assess the rate of spontaneous stabilization of DF treated with the frontline conservative approach in a single institute and to analyze the factors associated with spontaneous stabilization.

\section{METHODS}

\section{Patient Cohort}

We retrospectively reviewed 197 patients who were diagnosed as having sporadic extra-abdominal DF in our institute from 1995 to 2016. We conducted this study in compliance with the principles of the Declaration of Helsinki. The protocol of this study was reviewed and approved by the Institutional Review Board of Seoul National University Hospital (IRB No. 1610-008-795). The informed consent was waived. We included patients in whom (1) observation with a watchful waiting strategy was used as primary treatment, (2) regular follow-up with imaging modality was performed, and (3) follow-up period was more than 12 months. We excluded patients in whom (1) observation was less than 1 year, (2) medical or radiological information was insufficient to assess the extent of tumor, and (3) additional treatment such as chemotherapy and radiotherapy was required during conservative treatment. In total, 76 patients satisfied our inclusion criteria and were eligible for analysis.

\section{Tumor Surveillance}

Regular surveillance was performed with clinical examination and imaging modalities such as magnetic resonance imaging (MRI) and ultrasound. The extent of tumor was evaluated at the time of diagnosis and followed up with the same imaging modality every 3 to 6 months from the start of observation treatment except for four patients who were followed up with ultrasound. After the spontaneous stabilization of the disease, imaging assessment was continued with the same interval or annually. We defined stabilization of DF as radiological evidence of the size of the tumor unchanged or decreasing continuously for 6 months or more. The size of tumor was measured as the longest diameter of a measurable mass in any plane. Measurement was done by a radiologist (JYC) specializing in the musculoskeletal section and confirmed by three orthopedic oncologists (MSR, YK, and IH); in case of discrepancy, the three authors had a discussion and made a decision. During follow-up, date, symptoms including pain and neurological deficits, and tumor status including size and surveillance frequency were documented. The duration of our watchful waiting treatment was defined as the period from the date of diagnosis to the last follow-up unless the strategy was changed.

\section{Data Collection}

The following clinicopathological variables were collected from the institutional database: sex, age at first diagnosis, disease presentation, size and location of tumor, origin of tumor compartment, resection margin status, adjuvant chemotherapy, and adjuvant radiotherapy. The information is summarized in Table 1. Tumor location was dichotomized as axial or appendicular. The size of tumor was measured as the longest diameter of a measurable mass in any plane on MRI. Data on tumor size, compartment of origin, and tumor status at final follow-up were obtained from the MRI report written by the radiologist (JC) spe-

\section{Table 1. Demographic Characteristics}

\begin{tabular}{ll}
\hline \multicolumn{1}{|c}{ Variable } & Value (N = 76) \\
\hline Sex & $29(38.2)$ \\
\hline Male & $47(61.8)$ \\
\hline Female & \\
\hline Age (yr) & $48(63.2)$ \\
\hline$<40$ & $28(36.8)$ \\
\hline$\geq 40$ & \\
\hline Tumor size (cm) & $13(17.1)$ \\
\hline$<5$ & $62(81.6)$ \\
\hline$\geq 5$ & \\
\hline Tumor location & $37(48.7)$ \\
\hline Axial & $14(18.4)$ \\
\hline Upper extremity & $25(32.9)$ \\
\hline Lower extremity & $30(39.5)$ \\
\hline Compartment of origin & $26(34.2)$ \\
\hline Intramuscular & $46(60.5)$ \\
\hline Intermuscular & \\
\hline Disease presentation & \\
\hline Recurrent & \\
\hline
\end{tabular}

Values are presented as number (\%). 
Kim et al. Spontaneous Stabilization of Desmoid Fibromatosis

Clinics in Orthopedic Surgery • Vol. 12, No. 1, $2020 \bullet$ www.ecios.org

cializing in musculoskeletal oncology and were further confirmed in consensus by the three authors (MSR, YK, and $\mathrm{IH}$ ). Histology was confirmed by an experienced pathologist (CL). In patients with recurrence after previous surgical treatment outside of our institute, we reviewed the original slides from the previous hospital.

\section{Statistical Analysis}

The primary endpoint was spontaneous stabilization of DF. Time to stabilization was calculated as the interval between the date of diagnosis of DF and the date when stabilization was initially noted by imaging modality. For recurrent $\mathrm{DF}$, the time to stabilization was defined as the interval from the date of recurrence to the date when stabilization was confirmed. Evolving tumors were classified as censored at the last follow-up. Possible factors associated with disease stabilization of DF were analyzed with the Kaplan-Meier survival method and Cox proportional hazard method. Time to stabilization was estimated using the Kaplan-Meier survival method, and the log-rank test was used for univariate analysis. Multivariate analysis was performed by using the Cox proportional hazards model on variables in which $p$-values of $<0.05$ were obtained after univariate analysis. All statistical analyses were performed by using IBM SPSS ver. 21.0 (IBM Corp., Armonk, NY, USA). A two-tailed $p$-value of less than 0.05 was considered statistically significant.

\section{RESULTS}

\section{Stabilization Rate of DF}

Of the total 197 patients with DF, the initial treatment strategy was frontline observation in 107 patients and upfront surgery in 90 patients (Fig. 1). Of the 107 patients with observation, 47 patients were excluded from the analysis because of the follow-up of less than 1 year ( $n=$ 45) and changes in treatment strategy to chemotherapy or radiotherapy during observation $(\mathrm{n}=2)$. The observational therapy was continued to the final follow-up in 32 patients, whereas 28 patients underwent palliative surgery during the observational period (mostly because of neurologic symptoms and severe pain caused by mass effect). Among the 28 patients, seven were cured after complete excision without recurrence, whereas 21 patients had recurrence. Of the 21 patients, seven had additional chemotherapy or radiotherapy, and two continued to have surgical treatment; the remaining 12 had observational treatment after surgery and thus were included in the analysis. Of the 90 patients who underwent upfront surgery, 28 patients were excluded from the analysis: 27 with continuous disease-free status after surgery until the last follow-up ( $\mathrm{n}=27)$ and 1 with incomplete medical record $(\mathrm{n}=1)$. The remaining 62 patients had recurrence after surgical intervention. Of those, 21 had repetitive surgery and 9 had additional chemotherapy or radiotherapy; the remaining 32 underwent observational treatment until the last follow-up. In total, 76 patients satisfied our inclusion

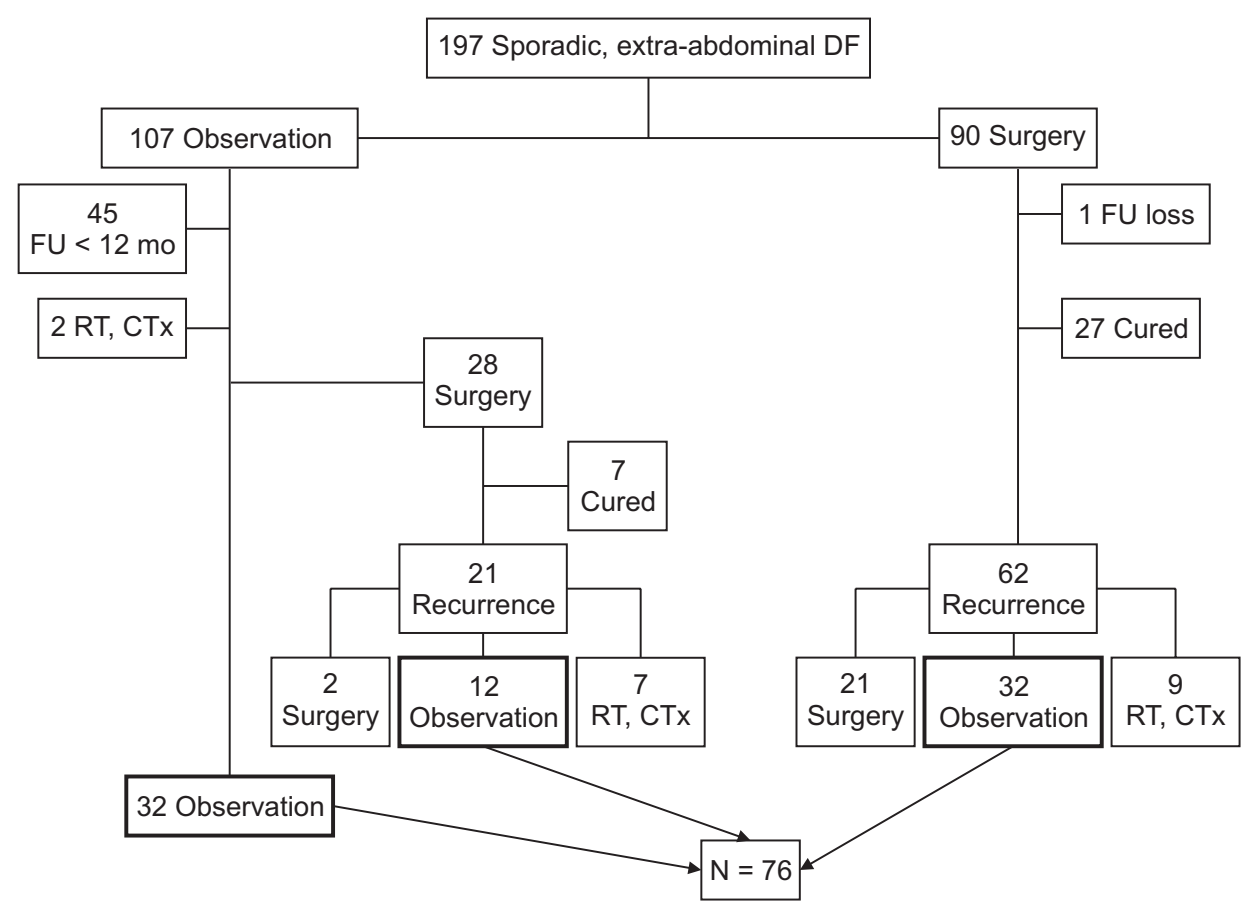

Fig. 1. Algorithm of patient selection. DF: desmoid-type fibromatosis, FU: follow-up, $\mathrm{RT}$ : radiation therapy, CTx: chemotherapy. 
Kim et al. Spontaneous Stabilization of Desmoid Fibromatosis

Clinics in Orthopedic Surgery • Vol. 12, No. 1, $2020 \bullet$ www.ecios.org

criteria and were included in the analysis.

On patient demographic characteristics (Table 1) of the 76 included patients, 29 (38.2\%) were men and 47 $(61.8 \%)$ were women. The mean age of the patients was 30.2 years (range, 1 to 77 years), and 48 patients (63.2\%) were younger than 40 years. Sixty-two patients (81.6\%) had tumors larger than $5 \mathrm{~cm}$. The location of the tumors was axial in 37 patients (48.7\%) and appendicular in 39 (51.3\%). Forty-six patients (60.5\%) had intermuscular tumors regardless of the depth, and $26(34.2 \%)$ had intramuscular tumors. The tumor was recurrent in 46 patients $(60.5 \%)$ at the time of presentation, whereas it was primary in 30 patients (39.5\%). The mean follow-up from the start of observation was 54.8 months (range, 12 to 226 months). Fifty-four (71.0\%) of 76 tumors showed spontaneous stabilization during observation treatment and were stable at the final follow-up. The mean time to stabilization was 30.4 months (range, 7 to 112 months). On Kaplan-Meier survival analysis, the spontaneous stabilization rate was $25.4 \%$ at 1 year, $52.7 \%$ at 2 years, and $70.9 \%$ at 3 years.

\section{Predictors of Disease Stabilization}

On univariate analysis, age and disease presentation were associated with stabilization of DF (Table 2). Patients who were younger than 40 years $(p=0.014)$ or had recurrence $(p=0.036)$ needed longer time for spontaneous stabiliza-

\begin{tabular}{|c|c|c|c|}
\hline Variable & $\begin{array}{l}\text { Hazard } \\
\text { ratio }\end{array}$ & $\begin{array}{l}95 \% \text { Confidence } \\
\text { interval }\end{array}$ & $p$-value \\
\hline \multicolumn{4}{|l|}{ Age (yr) } \\
\hline$<40$ & 2.04 & $1.12-3.73$ & 0.022 \\
\hline \multicolumn{4}{|l|}{$\geq 40$} \\
\hline \multicolumn{4}{|c|}{ Disease presentation } \\
\hline Recurrent & 1.79 & $1.03-3.09$ & 0.041 \\
\hline Primary & & & \\
\hline
\end{tabular}

\section{Table 2. Univariate Analysis of Factors Associated with Spontaneous Stabilization}

\begin{tabular}{|c|c|c|c|c|c|}
\hline Variable & Total (N) & No. of stable tumors & $\begin{array}{c}\text { Time to } \\
\text { stabilization (mo) }\end{array}$ & $\begin{array}{l}95 \% \text { Confidence } \\
\text { interval }\end{array}$ & $\begin{array}{c}p \text {-value } \\
\text { (log-rank) }\end{array}$ \\
\hline Sex & & & & & 0.674 \\
\hline Male & 29 & 22 & 33.6 & $22.2-45.0$ & \\
\hline Female & 47 & 32 & 29.7 & $21.7-37.6$ & \\
\hline Age (yr) & & & & & 0.023 \\
\hline$<40$ & 48 & 34 & 36.6 & $27.4-45.9$ & \\
\hline$\geq 40$ & 28 & 20 & 20.8 & $15.9-27.8$ & \\
\hline Tumor size (cm) & & & & & 0.887 \\
\hline$<5$ & 13 & 8 & 20.5 & $11.4-29.5$ & \\
\hline$\geq 5$ & 62 & 45 & 33.6 & $26.0-41.2$ & \\
\hline Tumor location & & & & & 0.148 \\
\hline Axial & 37 & 28 & 27.5 & $19.0-35.9$ & \\
\hline Extremity & 39 & 26 & 35.5 & $25.3-45.7$ & \\
\hline Compartment of origin & & & & & 0.299 \\
\hline Intramuscular & 26 & 19 & 28.0 & $17.7-38.2$ & \\
\hline Intermuscular & 46 & 32 & 33.4 & $24.3-42.4$ & \\
\hline Disease presentation & & & & & 0.023 \\
\hline Recurrent & 46 & 30 & 37.0 & $28.2-45.8$ & \\
\hline Primary & 30 & 24 & 24.7 & $15.1-34.3$ & \\
\hline
\end{tabular}


Kim et al. Spontaneous Stabilization of Desmoid Fibromatosis

Clinics in Orthopedic Surgery • Vol. 12, No. 1, $2020 \bullet$ www.ecios.org

tion. Tumor size had no correlation with spontaneous stabilization $(p=0.887)$. On multivariate analysis with the Cox proportional hazard method, age younger than 40 years (hazard ratio $[\mathrm{HR}], 2.04 ; p=0.022$ ) and recurrence (HR, $1.79 ; p=0.041$ ) were significant predictive factors of longer time to disease stabilization (Table 3 ).

\section{DISCUSSION}

DF is a benign, nonmetastasizing neoplasm marked by monoclonal fibroblastic proliferation of spindle cells with a locally infiltrative growth pattern. ${ }^{2)}$ Although this neoplasm is considered intermediately malignant because of its inability to metastasize, its aggressive nature is mainly reflected in the high rate of local recurrence, ${ }^{5-11,21-23)}$ which often leads to repetitive operations and entails substantial morbidity. ${ }^{24-27)}$ After publication of many reports on spontaneous disease stabilization or even regression without treatment, the shift from early proactive treatment approach to conservative "wait-and-see" approach has emerged. ${ }^{4,5,18,19)}$ In this study, we confirmed the validity of this recent approach by assessing the spontaneous stabilization rate of DF and evaluating predictive factors.

The spontaneous stabilization rate of DF after nonsurgical treatment varies from $50 \%$ to $97 \%$ in previous reports with a mean follow-up of 3 to 6 years. ${ }^{5,12,18,19,24)}$ The moderate stabilization rate $(71.0 \%)$ found in the present study falls within the described range. The potential of progression of DF seems to be limited after 3 years. ${ }^{5,12,18,19,24)}$ On the other hand, the mean time to tumor stabilization in the present study (30.4 months) is longer than that in a report by Barbier et al. (13.2 months). ${ }^{19)}$

In the present study, recurrence was found to be an independent predictor of spontaneous stabilization of DF as reported by Barbier et al. ${ }^{19)}$ Shin et al. ${ }^{28)}$ have also reported that recurrence is significantly associated with poor recurrence-free survival in their 119 patients treated with surgical resection. Huang et al. ${ }^{15)}$ also reported that patients with previous history of recurrence have shorter recurrence-free survival on the basis of the analysis of 214 patients who underwent surgical resection.

We found age younger than 40 years was associated with longer time to spontaneous stabilization of DF. Many studies ${ }^{7,11,23)}$ have also suggested that younger age be considered a predictive factor of shorter recurrence-free survival after surgical treatment. Therefore, age should be an important factor when a physician considers watchful waiting strategy in clinic.

Tumor size was variable and did not show significant correlation with stabilization in this study. Barbier et al. ${ }^{19)}$ retrospectively reviewed 26 cases of extra-abdominal $\mathrm{DF}$ and reported that the tumor size was very variable and independent of location and evolutivity. Even though tumor size is a well-known independent prognostic factor, ${ }^{9,15,23,29)}$ it did not appear to be predictive of the disease stabilization process. Fiore et al ${ }^{12)}$ reported that patients with tumors located in trunk or thoracic wall had worse progression-free survival. However, we found no significant correlation of the location of tumor with time to stabilization.

Among 54 patients whose tumor had stabilized, 13 patients showed transient spontaneous stabilization (the tumor size increased later). The increase in size was not significant in most patients. None of them required additional treatment and stayed with observation. The mean time to rebound was 31.3 months (range, 5 to 93 months). On the other hand, nine patients showed continuous shrinkage or decrease in tumor size. In particular, one patient with aggressive fibromatosis in the psoas muscle showed total regression during 15 months of observation. Further research on this issue is necessary.

The study is not without limitations. The shift towards a frontline conservative treatment approach is a relatively recent trend and surgical resection has been the gold standard for a long time. In our cohort, 90 patients had upfront surgery and $44(48 \%)$ of them had surgical treatment before 2010. Also, given the retrospective nature of our study, there were certain cases in which we could not identify the clinical reference to determine either surgical resection or a frontline conservative strategy. Measuring the proper size or volume of the tumors and evaluating the effect of treatment are another issue that needs to be addressed in further studies. In the current study, we used only the longest diameter of the measurable mass in any plane. In a recent study by Villalobos et al. ${ }^{30}$ ) on the long-term follow-up of the effect of PF03084014 of DF, they used both Response evaluation criteria in solid tumors criteria and World Health Organization criteria for assessment of the response.

Kasper et al. ${ }^{31)}$ suggested a consensus algorithm for the initial management of DF. According to the algorithm, there is a consensus that surgical treatment is no longer a gold standard for the initial treatment and the conservative "wait-and-see" approach should be the frontline treatment approach for patients with newly diagnosed DF, irrespective of symptoms. Our study supports this approach as an effective and reasonable initial option for the treatment of DF, and considering the identified predictive factors in treating DF can play a role in promoting disease stabilization. Frontline conservative treatment seems to be 
Kim et al. Spontaneous Stabilization of Desmoid Fibromatosis

Clinics in Orthopedic Surgery • Vol. 12, No. 1, $2020 \bullet$ www.ecios.org

the optimal treatment for most patients who present with DF. Younger patients or those with recurrence may require longer time to spontaneous stabilization of the neoplasm.

\section{CONFLICT OF INTEREST}

No potential conflict of interest relevant to this article was reported.

\section{ACKNOWLEDGEMENTS}

We thank Ja Young Choi, MD, Department of Radiology, Seoul National University Hospital, and Cheol Lee, MD, Department of Pathology, Seoul National Univeristy, for assistance with radiologic and pathologic insight and expertise that greatly improved the manuscript.

\section{REFERENCES}

1. MacFarlane J. Clinical reports of the surgical practice of the Glasgow Royal Infirmary. Glasgow: Robertson; 1832. 1:XVI314.

2. Otero S, Moskovic EC, Strauss DC, et al. Desmoid-type fibromatosis. Clin Radiol. 2015;70(9):1038-45.

3. Nieuwenhuis MH, Casparie M, Mathus-Vliegen LM, Dekkers OM, Hogendoorn PC, Vasen HF. A nation-wide study comparing sporadic and familial adenomatous polyposis-related desmoid-type fibromatoses. Int J Cancer. 2011;129(1):25661.

4. Stoeckle E, Coindre JM, Longy M, et al. A critical analysis of treatment strategies in desmoid tumours: a review of a series of 106 cases. Eur J Surg Oncol. 2009;35(2):129-34.

5. Bonvalot S, Eldweny H, Haddad V, et al. Extra-abdominal primary fibromatosis: Aggressive management could be avoided in a subgroup of patients. Eur J Surg Oncol. 2008;34(4):462-8.

6. Yoon GW, Kim JD, Chung SH. The analysis of treatment of aggressive fibromatosis using oral methotrexate chemotherapy. Clin Orthop Surg. 2014;6(4):439-42.

7. Peng PD, Hyder O, Mavros MN, et al. Management and recurrence patterns of desmoids tumors: a multi-institutional analysis of 211 patients. Ann Surg Oncol. 2012;19(13):403642.

8. Prodinger PM, Rechl H, Keller M, et al. Surgical resection and radiation therapy of desmoid tumours of the extremities: results of a supra-regional tumour centre. Int Orthop. 2013;37(10):1987-93.

9. Zeng WG, Zhou ZX, Liang JW, et al. Prognostic factors for desmoid tumor: a surgical series of 233 patients at a single institution. Tumour Biol. 2014;35(8):7513-21.

10. Wang YF, Guo W, Sun KK, et al. Postoperative recurrence of desmoid tumors: clinical and pathological perspectives. World J Surg Oncol. 2015;13:26.

11. Mueller C, Croner R, Klein P, Grutzmann R, Vassos N. Primary and recurrent sporadic desmoids: Prognostic factors influencing recurrence-free survival after complete gross resection. Int J Surg. 2016;31:63-70.

12. Fiore M, Rimareix F, Mariani L, et al. Desmoid-type fibromatosis: a front-line conservative approach to select patients for surgical treatment. Ann Surg Oncol. 2009;16(9):2587-93.

13. Bertani E, Testori A, Chiappa A, et al. Recurrence and prognostic factors in patients with aggressive fibromatosis: the role of radical surgery and its limitations. World J Surg Oncol. 2012;10:184.

14. Gronchi A, Colombo C, Le Pechoux C, et al. Sporadic desmoid-type fibromatosis: a stepwise approach to a non-metastasising neoplasm--a position paper from the Italian and the French Sarcoma Group. Ann Oncol. 2014;25(3):578-83.

15. Huang K, Wang CM, Chen JG, et al. Prognostic factors influencing event-free survival and treatments in desmoidtype fibromatosis: analysis from a large institution. Am J Surg. 2014;207(6):847-54.

16. Colombo C, Miceli R, Le Pechoux C, et al. Sporadic extra abdominal wall desmoid-type fibromatosis: surgical resection can be safely limited to a minority of patients. Eur J Cancer. 2015;51(2):186-92.

17. van Broekhoven DL, Verhoef C, Elias SG, et al. Local recurrence after surgery for primary extra-abdominal desmoidtype fibromatosis. Br J Surg. 2013;100(9):1214-9.

18. Mitchell G, Thomas JM, Harmer CL. Aggressive fibromatosis: evidence for a stable phase. Sarcoma. 1998;2(3-4):14954.

19. Barbier O, Anract P, Pluot E, et al. Primary or recurring extra-abdominal desmoid fibromatosis: assessment of treatment by observation only. Orthop Traumatol Surg Res. 2010;96(8):884-9.

20. Gronchi A, Raut CP. Optimal approach to sporadic desmoid tumors: from radical surgery to observation: time for a consensus? Ann Surg Oncol. 2012;19(13):3995-7.

21. Merchant NB, Lewis JJ, Woodruff JM, Leung DH, Brennan MF. Extremity and trunk desmoid tumors: a multifactorial 
Kim et al. Spontaneous Stabilization of Desmoid Fibromatosis

Clinics in Orthopedic Surgery • Vol. 12, No. 1, $2020 \bullet$ www.ecios.org

analysis of outcome. Cancer. 1999;86(10):2045-52.

22. Sri-Ram K, Haddo O, Dannawi Z, et al. The outcome of extra-abdominal fibromatosis treated at a tertiary referral centre. Eur J Surg Oncol. 2012;38(8):700-5.

23. He XD, Zhang YB, Wang L, et al. Prognostic factors for the recurrence of sporadic desmoid-type fibromatosis after macroscopically complete resection: Analysis of 114 patients at a single institution. Eur J Surg Oncol. 2015;41(8):1013-9.

24. Rock MG, Pritchard DJ, Reiman HM, Soule EH, Brewster RC. Extra-abdominal desmoid tumors. J Bone Joint Surg Am. 1984;66(9):1369-74.

25. Dalen BP, Bergh PM, Gunterberg BU. Desmoid tumors: a clinical review of 30 patients with more than 20 years' follow-up. Acta Orthop Scand. 2003;74(4):455-9.

26. Leithner A, Gapp M, Leithner K, et al. Margins in extraabdominal desmoid tumors: a comparative analysis. J Surg Oncol. 2004;86(3):152-6.

27. Lewis JJ, Boland PJ, Leung DH, Woodruff JM, Brennan MF. The enigma of desmoid tumors. Ann Surg. 1999;229(6):866-
72.

28. Shin SH, Ko KR, Cho SK, Choi YL, Seo SW. Surgical outcome of desmoid tumors: adjuvant radiotherapy delayed the recurrence, but did not affect long-term outcomes. J Surg Oncol. 2013;108(1):28-33.

29. Cates JM. Prognostic factors for second recurrence after surgical resection of recurrent desmoid-type fibromatosis. Pathol Oncol Res. 2015;21(4):1085-90.

30. Villalobos VM, Hall F, Jimeno A, et al. Long-term follow-up of desmoid fibromatosis treated with PF-03084014, an oral gamma secretase inhibitor. Ann Surg Oncol. 2018;25(3):76875.

31. Kasper B, Baumgarten C, Garcia J, et al . An update on the management of sporadic desmoid-type fibromatosis: a European Consensus Initiative between Sarcoma PAtients EuroNet (SPAEN) and European Organization for Research and Treatment of Cancer (EORTC)/Soft Tissue and Bone Sarcoma Group (STBSG). Ann Oncol. 2017;28(10):2399408. 